\title{
Randomized controlled trial comparing hyaluronic acid, platelet-rich plasma and the combination of both in the treatment of mild and moderate osteoarthritis of the knee
}

\author{
Lana JFSD ${ }^{1,4}$, Weglein $\mathrm{A}^{3}$, Sampson $\mathrm{S}^{2}$, Vicente $\mathrm{EF}^{1}$, Huber $\mathrm{SC}^{1,7}$, Souza $\mathrm{CV}^{4}$, Ambach $\mathrm{MA}^{5}$, Vincent $\mathrm{H}^{6}$, Urban-Paffaro $\mathrm{A}^{7}$, Onodera $\mathrm{CMK}^{7}$, \\ Annichino-Bizzacchi $\mathrm{JM}^{7}$, Santana $\mathrm{MHA}^{8}$, Belangero $\mathrm{WD}^{8}$
}

\begin{abstract}
Objective: This study aims at evaluating the clinical effects of Platelet Rich Plasma (PRP) and Hyaluronic Acid (HA) as individual treatments for mild to moderate Osteoarthritis (OA) and it also examines the potential synergistic effects of PRP in combination with HA. Research continues to emerge examining the potential therapeutic efficacy of HA and PRP as autologous injectable treatments for joint arthritis. However, there is a paucity of research investigating the effects of combining HA and PRP on pain and functional status in patients with OA.

Design: In this multi-center, randomized, controlled, double blind, prospective trial, 105 patients with mild to moderate knee osteoarthritis, who met the study criteria, were randomly allocated to one of three interventions: HA $(n=36)$, PRP $(n=36)$, or HA+PRP ( $n=33)$. Each patient received 3 intra-articular knee injections of their assigned substance, with 2 week intervals between each injection. Clinical outcomes were evaluated using the Western Ontario and McMaster Universities Arthritis Index (WOMAC) and Visual Analogue Scale (VAS) questionnaire at baseline and after 1,3,6 and 12 months.

Results: The study showed that the PRP group have significant reduction in VAS scores at $1(\mathrm{p}=0.003), 3(\mathrm{p}=0.0001), 6(\mathrm{p}=0.0001)$ and 12 $(\mathrm{p}=0.000)$ months when compared to HA. In addition, the PRP group illustrated greater improvement in WOMAC physical activity scale at 12 months $(\mathrm{p}=0.008)$ when compared to the HA group. Combining HA and PRP resulted in a significant decreases in pain ( $\mathrm{p}=0.0001)$ and functional limitation $(\mathrm{p}=0.0001)$ when compared to $\mathrm{HA}$ alone at 1 year post treatment; and significantly increased physical function at $1(\mathrm{p}=0.0004)$ and $3(\mathrm{p}=.011)$ months when compared to PRP alone.

Conclusion: The findings of the study support the use of autologous PRP as an effective treatment of mild to moderate knee osteoarthritis. It also shows that the combination of HA and PRP resulted to better outcomes than HA alone up to 1 year and PRP alone up to 3 months. Furthermore, the results suggest that combination of PRP and HA could potentially provide better functional outcomes in the first 30 days after treatment with both PRP and HA alone.
\end{abstract}

Key Words: Hyaluronic acid, Joint pathology, Knee, Osteoarthritis, Platelet-rich plasma

\section{Introduction}

Osteoarthritis of the knee joint has a great impact on physical performance and is considered one of the ten major causes of disability in the world. Standard conservative treatments for knee osteoarthritis include: weight loss, physical exercises, use of nonsteroid anti-inflammatory agents, analgesics, injection of hyaluronic acid (HA) and injection of glucocorticoids ${ }^{[1,2]}$. Although, standard conservative measures can provide symptomatic improvements, they are not without their limitations. Steroid injections are common practice among practitioners, including orthopedic surgeons, however, prolonged use of such pharmacological treatments may have adverse effects on existing cartilage $^{[3]}$. Also, chronic use of anti- inflammatory medications may cause nephrotoxicity and gastrointestinal side effects ${ }^{[3]}$. However, recently, Orthobiologic injections have emerged as a potentially safe and efficacious option for joint Osteoarthritis.

Hyaluronic Acid (HA) is currently a widely used injectable treatment for degenerative joint pathology. It is a glycosaminoglycan that acts as a backbone for proteoglycans of the extracellular matrix ${ }^{[4]}$, providing increased joint lubrication. Studies have demonstrated that HA has positive therapeutic efficacy for knee osteoarthritis with initial efficacy at 4 weeks, and peak effectiveness at 8 weeks which lasts for up to 6 months ${ }^{[5]}$. When compared to continuous oral NSAIDS or other anti-inflammatory medications, HA has illustrated comparable, if not greater, therapeutic effects on knee OA with a better safety profile ${ }^{[5,6]}$.

Autologous platelet rich plasma (PRP) has also emerged as an alternative in the context of injectable treatment for OA. PRP is comprised of a potent cellular milieu containing platelet concentrations above baseline, as well as an undifferentiated mixture of anti-inflammatory, pro-inflammatory, anabolic and catabolic mediators in an attempt to stimulate a supra-physiologic response and elicit the body's natural healing potential. Currently, most studies on PRP are anecdotal or case reports with small sample sizes. However, larger randomized controlled trials have demonstrated superior efficacy in areas such as tendinopathies ${ }^{[7]}$ and knee osteoarthritis $^{[8]}$.

\footnotetext{
Author Names in full: José F. S. D. Lana ${ }^{1,4}$, Adam Weglein ${ }^{3}$, Steve E. Sampson ${ }^{2}$, Eduardo F. Vicente ${ }^{1}$, Stephany Cares Huber ${ }^{1,7}$, Clarissa V. Souza ${ }^{4}$, Mary A. Ambach ${ }^{5}$, Hunter Vincent $^{6}$, Aline Urban-Paffaro ${ }^{7}$, Carolina M. K. Onodera ${ }^{7}$, Joyce M. Annichino-Bizzacchi ${ }^{7}$, Maria Helena A. Santana ${ }^{8}$, William D. Belangero ${ }^{4}$

${ }^{1}$ Bone and Cartilage Institute - (IOC) Indaiatuba - Brazil, ${ }^{2}$ David Geffen School of Medicine at UCLA - USA, ${ }^{3}$ Regenerative Ortho Med Clinic - USA

${ }^{4}$ Institute of Orthopedics and Traumatology of University of Campinas (UNICAMP) - Brazil , ${ }^{5}$ Orthohealing Center - USA, ${ }^{6}$ Department of Physical Medicine and Rehabilitation: UC Davis, ${ }^{7}$ Hemocentro of Campinas, University of Campinas (UNICAMP)- Brazil, ${ }^{8}$ School of Chemical Engineering University of Campinas (UNICAMP) - Brazil
} 
Although many studies have suggested both HA and PRP have potential to enhance the cartilage healing process and slow down the

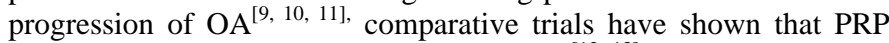
can be superior to $\mathrm{HA}$ in treating knee $\mathrm{OA}^{[12,13]}$. Furthermore, PRP with its potent mixture of growth factors and cytokines has also been shown to increase the production of HA from native synoviocytes $^{[14]}$. These findings suggest a potential additive effect of combining PRP with HA in treating OA. However, there is not much research examining such synergistic effects.

The main objective of our study was to evaluate the effectiveness of HA and PRP as monotherapies for mild to moderate OA and compare the results to the combination of PRP+HA.

\section{Materials and Methods}

\section{Research design}

The study is a double-blind, randomized and controlled prospective with three groups receiving three different lines of treatment. After the selection, the patients were randomized 1: 1: 1 by lot, in the three different treatment groups. The selection and randomization was performed by a biomedical team responsible for research. After review and approval by the institutional ethics committee, volunteer participants were blinded and subjected to a standardized injection protocol performed by one interventionist. The participants then completed the WOMAC and VAS questionnaires at baseline and at 30, 90, 180 and 360 days after treatment.

\section{Patients and sampling}

The study was conducted in two orthopedic clinics as well as one ambulatory regenerative medicine clinic in Brazil from January 2011 to April 2014. One hundred and twenty patients were enrolled in our study, but 105 (87.5\%) were ultimately included, as fifteen patients were lost for follow up after randomization. The local ethics committee of University of Uberaba approved the study (authorization number 0042.0.227.000-11) and all volunteer participants signed informed consent and were randomly allocated to one of the three groups of intervention, namely, HA, PRP, or $\mathrm{PRP}+\mathrm{HA}$ groups, as evidenced in the figure 1. The following inclusion criteria for patient selection were used: age between 40 to 70 years, history of chronic pain for at least four months and/or joint edema and radiographic evidence of mild to moderate OA according to Kellgren-Lawrence classification (grade I, II and III $)^{[15]}$. The exclusion criteria were considered to be: coagulopathies, axial deviation of lower limb larger than $5^{\circ}$ for valgus and varus knee, severe cardiovascular diseases, diabetes mellitus and, immunosuppressive status, patients on anticoagulants, antithrombotic and anti-platelet drugs and non-steroid antiinflammatory medication, patients with less than $11 \mathrm{mg} / \mathrm{dL}$ of hemoglobin and less than $150.000 \mathrm{~mm}^{3}$ platelets, auto-immune diseases, history of previous surgery in the affected joint and OA grade IV according to Kellgren-Lawrence classification. In addition, $\mathrm{C}$ reactive protein (CRP) and Uric acid levels were also assessed in all patients and abnormal levels were used as exclusion criteria.

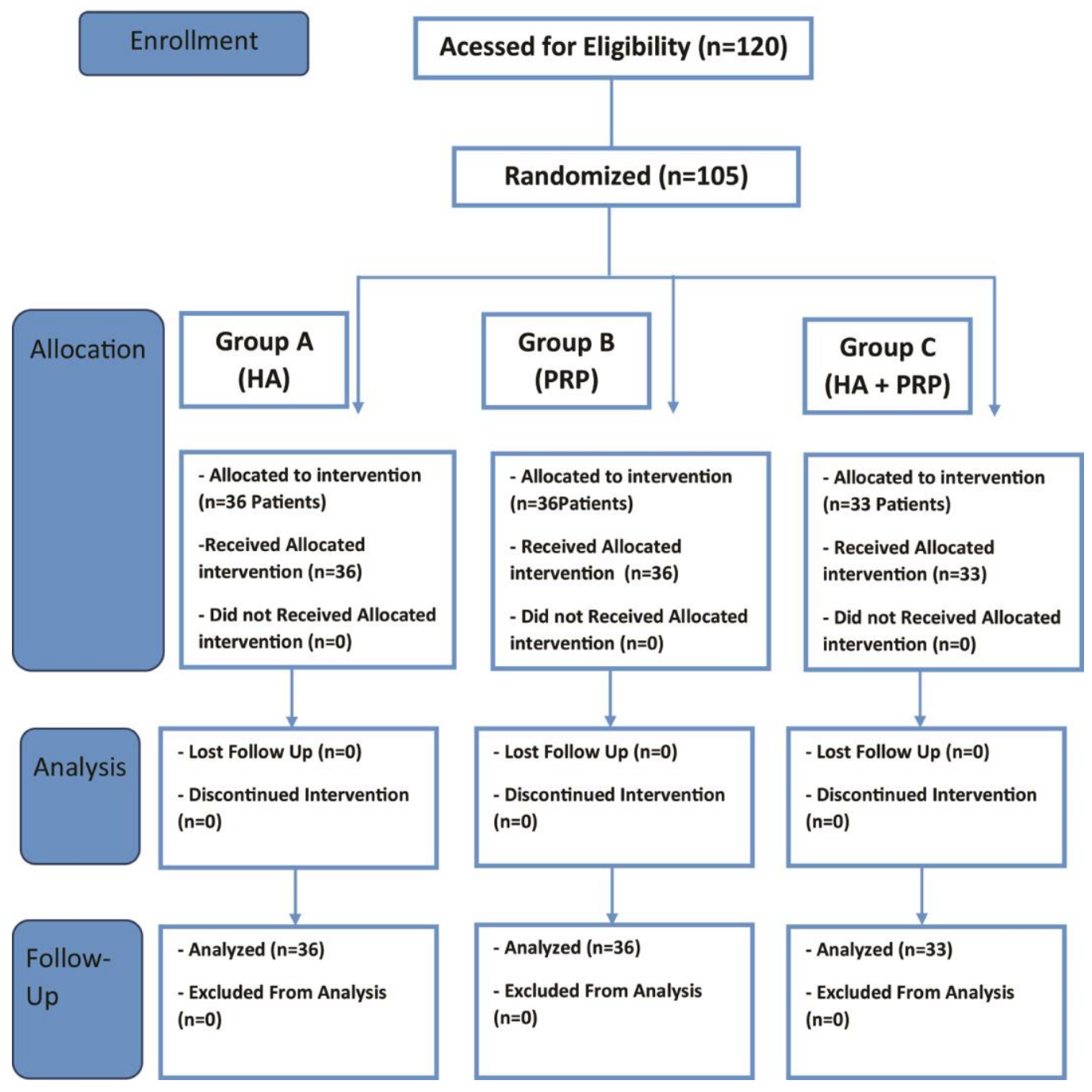

Figure 1: Flow-chart and details for all phases of research. 
In order to evaluate the systemic inflammation, the levels of CPR were measured in 30, 90, 180 and 360 days after the first application of PRP.

\section{Assessment and Outcomes}

Patients were assessed at screening for clinical and demographic characteristics and baseline assessment. Patients were followed for over one year and were submitted to four more follow-up evaluations: +30 (one month), +90 (3 months), +180 (6 months) and 360 days (1 year), where they completed VAS and WOMAC questionnaires. The version of the Western Ontario and McMaster Universities Arthritis Index (WOMAC) ${ }^{[16]}$ used in the study was translated and validated to Brazilian Portuguese ${ }^{[17]}$. The WOMAC scores are presented by Likert scale in which each question has a score ranging from 0 to 100 , distributed as follows: 0 $=$ none $; 25=$ little $; 50=$ Moderate $; 75=$ severe $; 100=$ very intense. Each of the five scores is calculated as the sum of the items included $^{[18]}$.

The primary analysis is the median change in VAS and WOMAC scores from pre-treatment baseline to 360 days across the three groups. Since VAS and WOMAC outcomes did not follow normal distribution, median values were used. To control for baseline differences, we subtracted the baseline and compared changes from baseline. This makes for a more fair comparison where differences are not due to differences at baseline. The secondary analysis is the median change in VAS and WOMAC scores from pre-treatment baseline to 30,90 and 180 days.

\section{Intervention}

Patients were asked to discontinue the use of any antiinflammatory drugs two weeks before initiation of treatment until trial completion. The procedure was performed in a procedure room at a clinic setting. Ultrasound guidance at knee medial compartment in $30^{\circ}$ degree of flexion was used in the procedure.

Injections were performed under ultrasound guidance three times in the affected knee(s), with interval of two weeks between them. The injections were administered using the lateral mid-patellar approach using strict sterile technique. Lidocaine $2 \%$ with epinephrine was used for local anesthesia (without intra articular anesthesia), buffering with $0,2 \mathrm{ml}$ of sodium bicarbonate $(8.4 \%)$. The PRP group received $5 \mathrm{ml}$ of platelet rich plasma (white blood cells (WBC) rich, red blood cells (RBC) poor, activated with serum), while the HA group received $2.0 \mathrm{ml}(20 \mathrm{mg}$ of $\mathrm{HA})$ of high molecular weight $(2.4-3.6$ million daltons) non cross-linked hyaluronic acid extracted from bacteria cells (Eufflexa-Ferring 10mg/ml HA). The PRP+HA group received both treatments, with the $2.0 \mathrm{ml}$ injection of HA first, followed by the $5 \mathrm{ml}$ of PRP. All the patients treated with PRP alone or in combination of HA used the same volume of PRP which is $5 \mathrm{ml}$. After the injections, patients were instructed to apply local icepack, three times a day for 30 minutes each in the first 2 days after injection and switch to hot packs in the third and fourth days after injection. Patients took Dipirone $1.0 \mathrm{~g}$ twice a day for the first two days after procedure.

\section{PRP Preparation}

Blood was collected from the patients after a fasting period of four hours. About $60 \mathrm{ml}$ of total blood was drawn under aseptic conditions, primarily from the median or antecubital vein. Basal platelet count was performed and the PRP was collected. $8.5 \mathrm{ml}$ of the anticoagulant ACD (citric acid, sodium citrate, dextrose) was used to preserve the blood cells and to maintain physiologic $\mathrm{pH}^{[19,20]}$.
The first centrifugation was carried out at (300G for 5 minutes), with the purpose of separating the blood components into three main layers: red blood cells (RBC) or erythrocytes, white blood cells (WBC) or buffy coat and plasma. Studies under way at UNICAMP University of Campinas, Brazil, suggest that at this speed there is a better recuperation of the platelets contained in the sample ${ }^{[21]}$. The whole top part of the content of the tubes (plasma and buffy coat) is collected, avoiding the collection of erythrocytes ${ }^{[22]}$. This content continues on to the second centrifugation at a higher speed rotation (700G for 17 minutes), which will promote a higher sedimentation of platelets and leukocytes. In this manner, it is possible to obtain a higher concentration of platelets in the final product, with no alteration to its integrity and causing no harm in the liberation of the platelet growth factors ${ }^{[23,24]}$. Platelet count was performed pre and post centrifugation in a hematological counter (ABX Micro 60-OT, Horiba $\mathrm{ABX}$ ) to ensure the highest level of quality control.

Activation is carried out with only autologous thrombin, in the proportion of $0.8 \mathrm{ml}$ of thrombin for $5 \mathrm{ml}$ of PRP.

\section{Statistical Analyses}

Descriptive analyses are provided for demographic and clinical characteristics. The Kolmogorov-Smirnov and Shapiro-Wilk tests were applied to check distributions for normality. Chi-square tests were used to compare binary outcomes and the, Kruskall-Wallis and Wilcoxon tests were applied to compare distributions of continuous data The primary analysis is the median change in VAS and WOMAC scores from pre-treatment baseline to 360 days across the three groups. A two-sided $\mathrm{p}$ value less than 0.05 was considered statistically significant. Computation were carried out using IBM SPSS v 21 and SAS versión 9.4.

\section{Results}

The mean age was 60.9 years (45-70), 90 patients $(84.8 \%)$ were female. In all of the 105 patients, a mild adverse reaction in the form of a knee swelling was reported 3-5 days after the application. It was not a reported as a major complication by any patient. It was verified that the majority of patients in all the groups have grade II of OA by Kellgren-Lawrence in the right knee. Also, it was observed that the patients were overweight through BMI in all the groups of treatment. The majority of the population was brown and has as comorbidities hypothyroidism, dyslipidemia and hypertension. Half of the patients in all the groups were physically active, practicing walking or aquatic activities, without axial impact. These data were presented in Table 1. It was found that statistical differences in the groups related to race $(\mathrm{p}=0.0076)$ and comorbidities $(p=0.0266)$. This difference in race was observed in the group treated with association of $\mathrm{PRP}+\mathrm{HA}$ in comparison to $\mathrm{HA}$ $(p=0.0201)$ and PRP $(p=0.0246)$. In regard to the comorbidities, a significant difference was observed between the groups that were treated with HA+PRP and PRP ( $\mathrm{p}=0.0031)$.

In the baseline only WOMAC pain were significant (HA-PRP $p=0.0073 ; \mathrm{HA}+\mathrm{PRP}-\mathrm{PRP} \mathrm{p}=0.0165)$, evidenced that the group of HA present more pain than the other two groups, as verified in Table 2. Three days after the treatment, it was verified thru VAS that the HA groups continued with significant more pain than the other groups (HA-PRP $\mathrm{p}=0.0034$; HA+PRP-PRP $\mathrm{p}=0.0113$ ). It was also observed that there was a significant improvement on the WOMAC physical in the group treated with HA+PRP when compared to the other groups (HA+PRP-HA p=0.0001; HA+PRP-PRP $\mathrm{p}=0.0004)$, as described in Table 3. According to the VAS, in 90 days, it was verified that the groups treated with PRP alone $(p=0.0001)$ or in combination $(\mathrm{p}=0.0000)$ showed significant less pain than HA. Also 
an improvement in WOMAC physical was observed in the group HA+PRP when compared with the other groups (HA- HA+PRP $\mathrm{p}=0.0052$; $\mathrm{PRP}-\mathrm{HA}+\mathrm{PRP} \mathrm{P}=0.0110$ ) as reported in Table 4. At the 180 day mark, significantly less pain was observed in the groups treated with PRP alone or in the combination and an improvement in the WOMAC physical was observed only for the group HA+PRP in comparison with HA ( $\mathrm{p}=0.0262)$, as observed in the Table 5. This tendency was verified at 360 days, as demonstrated in Table 6 . It was verified that the groups treated with PRP alone $(p=0.0000)$ or in combination ( $\mathrm{p}=0.0000)$ showed significant less pain in comparison to HA according to VAS. Also, these groups showed a significant improvement in WOMAC physical in comparison to HA (HA+PRPHA $\mathrm{p}=0.0001$; PRP-HA $\mathrm{p}=0.0089$ ).

In summary, the PRP group had significantly greater median VAS improvement at 30, 90, 180, 360 days and significantly greater WOMAC PA improvement at 360 days when compared to the HA group. The HA + PRP group had statistically significant decrease from baseline of the median VAS and WOMAC PA when compared to the HA group. Thus, combining HA and PRP resulted to less pain and less functional limitation compared to HA alone at 30, 90, 180 and 360 days. When comparing HA + PRP group to PRP alone, the combination resulted in a statistically significant improvement in median WOMAC PA values at 30 and 90 days only. Figures 3 and 4 summarizes the median VAS and WOMAC PA change from baseline, respectively. The median changes from day 0 baseline in WOMAC pain and WOMAC stiffness were not statistically different among the three groups at any time.

Relative to PRP, our final PRP product consisted of platelets, leukocytes and circulating fibrinogen, with a small residue of red cells (Figure 2). The PRP platelet concentration varied between 800,000 and $1,600,000$ per $\mathrm{mm}^{3}$ of plasma, which corresponds to 5 to 8 times the basal concentration in all patients. The basal concentration of platelets was 155.000 to $315.000 \mathrm{~mm}^{3}$ and white blood cells (WBC) were $9.7 \pm 3.4$ per $\mathrm{mm}^{3}$.

In relation to the inflammation, the level of CPR showed evidence that the group treated with PRP alone or in association had an increase in the CPR levels after 90 days and a decrease at the end of the follow-up. On the other hand, the group treated with HA showed an increase in 30 days with a posterior decrease in 90 days, but at the end of follow-up the levels of CPR were higher than baseline, as verified in the Table 7. In baseline, PRP group had the highest CPR value, being significant in comparison of $\mathrm{HA}+\mathrm{PRP}$ and $\mathrm{HA}$ $(\mathrm{p}=0.0013) .30$ days after the first application, HA group showed the highest value of CPR in comparison to other groups $(p=0.0152)$. In 90 days, it was observed that the HA group had the lowest value when compared with other groups of PRP alone or in association $(\mathrm{p}<0.0001)$. In 180 and 360 days after treatment it was verified the same pattern. Patients treated with HA presented highest values of $\mathrm{CPR}$ in comparison to the groups treated with PRP $(\mathrm{p}<0.001)$.

Table 1: Patients Casuistic $(\mathrm{n}=105)^{*}$

\begin{tabular}{|c|c|c|c|}
\hline & HA group $(n=36)$ & PRP group $(n=36)$ & $\underline{\text { HA+PRP group }(n=33)}$ \\
\hline Sex, F:M, (\%) & 33 (91.7): $3(8.3)$ & 29(80.6): 7(19.4) & 27 (81.8): 6 (18.2) \\
\hline Age, mean \pm SD (range) & $60 \pm 6.6(45-70)$ & $60.9 \pm 7(48-70)$ & $62 \pm 6.1(48-70)$ \\
\hline $\begin{array}{l}\text { Kellgren-Laurence grade, } \\
1: 2: 3(\%)\end{array}$ & $9(25): 16(44): 11(31)$ & $9(25): 14(39): 13(36)$ & $5(15.2): 14(42.4): 14(42.4)$ \\
\hline Limb, R:L:both (\%) & 17(47.2):13(6.1):6(16.7) & 14(39):16(44):5(15) & 21(64):7(21):5(15) \\
\hline BMI & $28.24 \pm 8.77$ & $27.42 \pm 6.89$ & $29.15 \pm 7.31$ \\
\hline Caucasian & $14(38.9 \%)$ & $8(22.2 \%)$ & $4(12.1 \%)$ \\
\hline Brown & $19(52.8 \%)$ & $27(75 \%)$ & $21(63.6 \%)$ \\
\hline Black & $3(8.3 \%)$ & $1(2.8 \%)$ & $8(24.2 \%)$ \\
\hline Hypothyroidism & $4(11.1 \%)$ & $3(8.3 \%)$ & $6(18.2 \%)$ \\
\hline Dyslipidemia & $12(33.3 \%)$ & $12(33.3 \%)$ & $8(24.2 \%)$ \\
\hline HT & $15(41.7 \%)$ & $11(30.6 \%)$ & $19(57.6 \%)$ \\
\hline $\begin{array}{l}\text { Physical Activity (walk or } \\
\text { aquatic activity without } \\
\text { axial impact, up until } 3 x \\
\text { per week) }\end{array}$ & $16(44.4 \%)$ & $23(63.8 \%)$ & $17(51.5 \%)$ \\
\hline
\end{tabular}

* P statistically significant in race $(\mathrm{p}=0.0076)$ and comorbidities $(\mathrm{p}=0.0266)$.

$\mathrm{HA}=$ hyaluronic acid; $\mathrm{PRP}=$ platelet-rich plasma; $\mathrm{WBC}=$ white blood cells; $\mathrm{F}=$ female; $\mathrm{M}=$ male $\mathrm{SD}=$ standard deviation; $\mathrm{R}=$ right; $\mathrm{L}=$ left $; \mathrm{BMI}=$ body mass index; $\mathrm{HT}=$ hypertension 
Table 2: Baseline comparisons of VAS, WOMAC Pain, WOMAC stiffness and WOMAC physical activity (PA). Median (range) reported.

\begin{tabular}{|c|c|c|c|c|c|c|}
\hline \multirow{3}{*}{ Outcome } & \multirow{3}{*}{ HA $(n=36)$} & \multirow{3}{*}{$\operatorname{PRP}(n=36)$} & \multirow{3}{*}{$\mathrm{HA}+\mathrm{PRP}(\mathrm{n}=33)$} & \multicolumn{3}{|c|}{$P$ values } \\
\hline & & & & HA vs & HA $v s$ & PRP vs \\
\hline & & & & PRP & HA+PRP & HA+PRP \\
\hline VAS & $7.0(5-10)$ & $7.5(3-10)$ & $7.0(5-10)$ & 0.2100 & 0.1447 & 0.7680 \\
\hline WOMAC & $388(125-500)$ & $288(100-500)$ & $275(100-500)$ & 0.0173 & 0.0165 & 0.5662 \\
\hline \multicolumn{7}{|l|}{ Pain } \\
\hline WOMAC & $75(25-200)$ & $125(25-200)$ & $125(25-200)$ & 0.0972 & 0.0582 & 0.5278 \\
\hline \multicolumn{7}{|l|}{ Stiffness } \\
\hline WOMAC & $988(425-1425)$ & $913(425-1425)$ & $950(450-1425)$ & 0.1993 & 0.5954 & 0.3282 \\
\hline PA & & & & & & \\
\hline
\end{tabular}

Table 3: Median change from base (pre) to 30 days. Median (range) reported.

\begin{tabular}{|c|c|c|c|c|c|c|}
\hline \multirow{3}{*}{ Outcome } & \multirow{3}{*}{ HA } & \multirow{3}{*}{ PRP } & \multirow{3}{*}{ HA+PRP } & \multicolumn{3}{|c|}{$P$ values } \\
\hline & & & & HA $v s$ & HA vs & \\
\hline & & & & PRP & HA+PRP & HA+PRP \\
\hline VAS & $-3.0(-7,0)$ & $-4.5(-8,0)$ & $-4.0(-8,-1)$ & 0.0034 & 0.0113 & 0.9417 \\
\hline $\mathrm{n}$ & 35 & 36 & 33 & & & \\
\hline WOMAC Pain & $-200(-450,25)$ & $-175(-350,75)$ & $-175(-375,25)$ & 0.1420 & 0.1191 & 0.8355 \\
\hline $\mathrm{n}$ & 36 & 34 & 33 & & & \\
\hline \multirow{2}{*}{$\begin{array}{l}\text { WOMAC Stiffness } \\
\mathrm{n}\end{array}$} & $-50.0(-175,0)$ & $-50.0(-150,25)$ & $-75(-100,-25)$ & 0.7900 & 0.1028 & 0.2334 \\
\hline & 23 & 22 & 19 & & & \\
\hline WOMAC PA & $-362.5(-1075,200)$ & $-375.0(-1050,275)$ & $-650(-1125,-75)$ & 0.1909 & 0.0001 & 0.0004 \\
\hline $\mathrm{n}$ & 36 & 36 & 33 & & & \\
\hline
\end{tabular}

Table 4: Median change from base (pre) to 90 days. Median (range) reported.

\begin{tabular}{|c|c|c|c|c|c|c|}
\hline \multirow{3}{*}{ Outcome } & \multirow{3}{*}{ HA } & \multirow{3}{*}{ PRP } & \multirow{3}{*}{ HA+PRP } & \multicolumn{3}{|c|}{$P$ values } \\
\hline & & & & HA vs & HA vs & PRP vs \\
\hline & & & & PRP & HA+PRP & HA+PRP \\
\hline VAS & $-3.0(-6,0)$ & $-6.0(-8,1)$ & $-6.0(-9,-1)$ & 0.0001 & 0.0000 & 0.1795 \\
\hline $\mathrm{n}$ & 36 & 35 & 33 & & & \\
\hline WOMAC Pain & $-187.5(-450,75)$ & $-225.0(-375,0)$ & $-200(-375,-25)$ & 0.5113 & 0.2652 & 0.6617 \\
\hline $\mathrm{n}$ & 36 & 33 & 33 & & & \\
\hline WOMAC & $-50.0(-125,25)$ & $-100.0(-175,0)$ & $-75.0(-125,-25)$ & 0.1282 & 0.1382 & 0.7284 \\
\hline $\begin{array}{l}\text { Stiffness } \\
\mathrm{n}\end{array}$ & 22 & 22 & 19 & & & \\
\hline WOMAC PA & $-512.5(-1225,500)$ & $-550.0(-1150,25)$ & $-725.0(-1225,-25)$ & 0.4368 & 0.0052 & 0.0110 \\
\hline $\mathrm{n}$ & 36 & 36 & 33 & & & \\
\hline
\end{tabular}


Table 5: Median change from base (pre) to 180 days. Median (range) reported.

\begin{tabular}{|c|c|c|c|c|c|c|}
\hline \multirow{3}{*}{ Outcome } & \multirow{3}{*}{ HA } & \multirow{3}{*}{ PRP } & \multirow{3}{*}{ HA+PRP } & \multicolumn{3}{|c|}{$P$ values } \\
\hline & & & & HA $v s$ & HA $v s$ & PRP vs \\
\hline & & & & PRP & HA+PRP & HA+PRP \\
\hline VAS & $-3.0(-7,4)$ & $-5.0(-9,-1)$ & $-5.0(-9,-1)$ & 0.0001 & 0.0000 & 0.2235 \\
\hline $\mathrm{n}$ & 36 & 35 & 33 & & & \\
\hline WOMAC Pain & $-162.5(-450,250)$ & $-225.0(-450,0)$ & $-200(-450,-25)$ & 0.1555 & 0.3029 & 0.5579 \\
\hline $\mathrm{n}$ & 36 & 34 & 33 & & & \\
\hline WOMAC & $-62.5(-125,0)$ & $-62.5(-125,0)$ & $-100(-150,25)$ & 0.9226 & 0.0953 & 0.0698 \\
\hline Stiffness & 14 & 18 & 14 & & & \\
\hline $\mathrm{n}$ & & & & & & \\
\hline WOMAC PA & $-462.5(-1350,600)$ & $-625.0(-1400,0)$ & $-675.0(-1300,-250)$ & 0.0909 & 0.0262 & 0.1629 \\
\hline $\mathrm{n}$ & 36 & 36 & 33 & & & \\
\hline
\end{tabular}

Table 6: Comparison of median VAS, WOMAC pain, WOMAC stiffness and WOMAC physical activity (PA) change from baseline to 360 days. Median (range) reported

\begin{tabular}{|c|c|c|c|c|c|c|}
\hline \multirow{3}{*}{ Outcome } & \multirow{3}{*}{ HA } & \multirow{3}{*}{ PRP } & \multirow{3}{*}{ HA+PRP } & \multicolumn{3}{|c|}{$P$ values } \\
\hline & & & & HA vs & HA $v s$ & PRP vs \\
\hline & & & & PRP & HA+PRP & HA+PRP \\
\hline VAS & $-2(-7,2)$ & $-5(-9,1)$ & $-5(-9,-1)$ & 0.0000 & 0.0000 & 0.6783 \\
\hline $\mathrm{n}$ & 33 & 32 & 25 & & & \\
\hline WOMAC Pain & $-188(-450,50)$ & $-238(-425,0)$ & $-200(-450,50)$ & 0.2273 & 0.6546 & 0.3057 \\
\hline $\mathrm{n}$ & 34 & 30 & 33 & & & \\
\hline WOMAC & $-75(-125,50)$ & $-100(-175,0)$ & $-88(-175,0)$ & 0.3192 & 0.4674 & 0.7537 \\
\hline Stiffness & 15 & 14 & 12 & & & \\
\hline $\mathrm{n}$ & & & & & & \\
\hline WOMAC PA & $-450(-1350,375)$ & $-775(-1300,0)$ & $-825(-1325,-300)$ & 0.0089 & 0.0001 & 0.1982 \\
\hline $\mathrm{n}$ & 36 & 34 & 33 & & & \\
\hline
\end{tabular}

Table 7: Results of mean and standard deviation of CPR levels of different groups in the follow-up

\begin{tabular}{lllll} 
CPR levels & HA & PRP & HA+PRP & P values \\
\hline Baseline & $2.6 \pm 1.7$ & $3.3 \pm 2.3$ & $2.6 \pm 1.6$ & 0.0013 \\
\hline+30 & $4.7 \pm 1.7$ & $3.6 \pm 1.3$ & $3.8 \pm 1.4$ & 0.0152 \\
\hline+90 & $2.6 \pm 1.4$ & $4.4 \pm 1.4$ & $4.2 \pm 1.6$ & $<0.0001$ \\
\hline+180 & $6.0 \pm 2.3$ & $3.1 \pm 1.6$ & $3.4 \pm 1.6$ & $<0.0001$ \\
\hline+360 & $6.3 \pm 2.3$ & $2.8 \pm 1.4$ & $3.0 \pm 1.4$ & $<0.0001$ \\
\hline
\end{tabular}




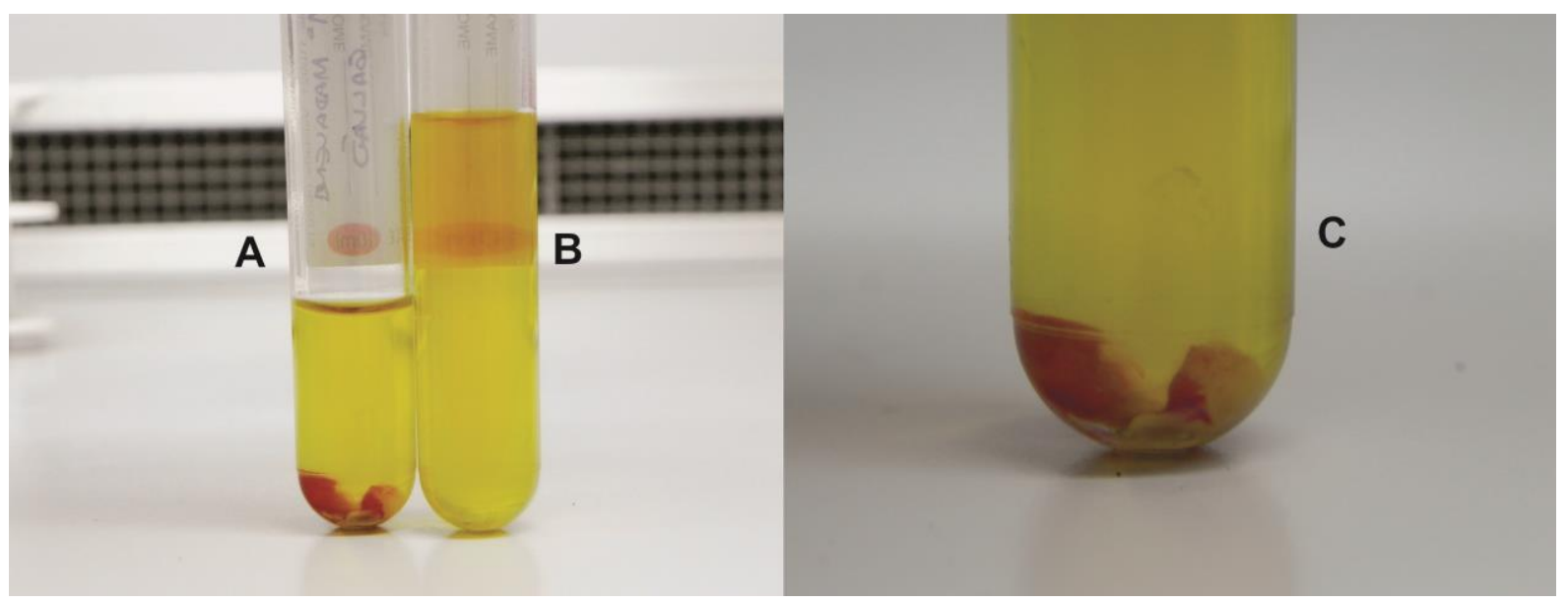

Figure 2: (A) Platelet-Rich Plasma and leukocytes (L-PRP) containing the white series (buffy coat) and residual red cells; (B) top part of the collected plasma after the second centrifugation, which corresponds to the platelet poor plasma (PPP), approximately $80 \%$ of the total centrifuged volume; C) Cellular fraction in evidence before PRP suspension.

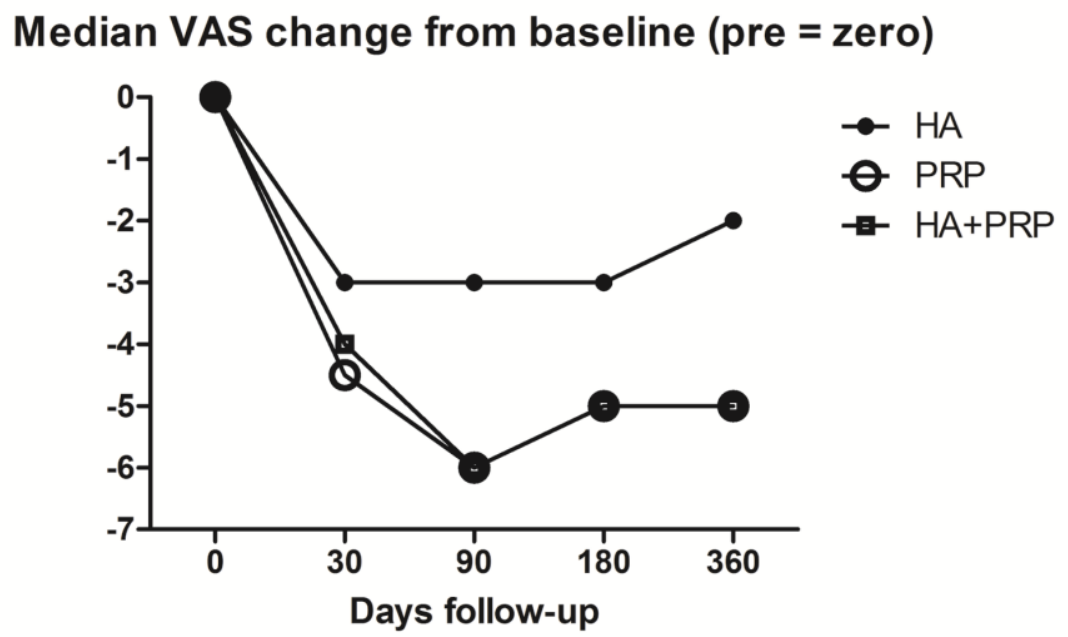

Figure 3: Median VAS change from Baseline

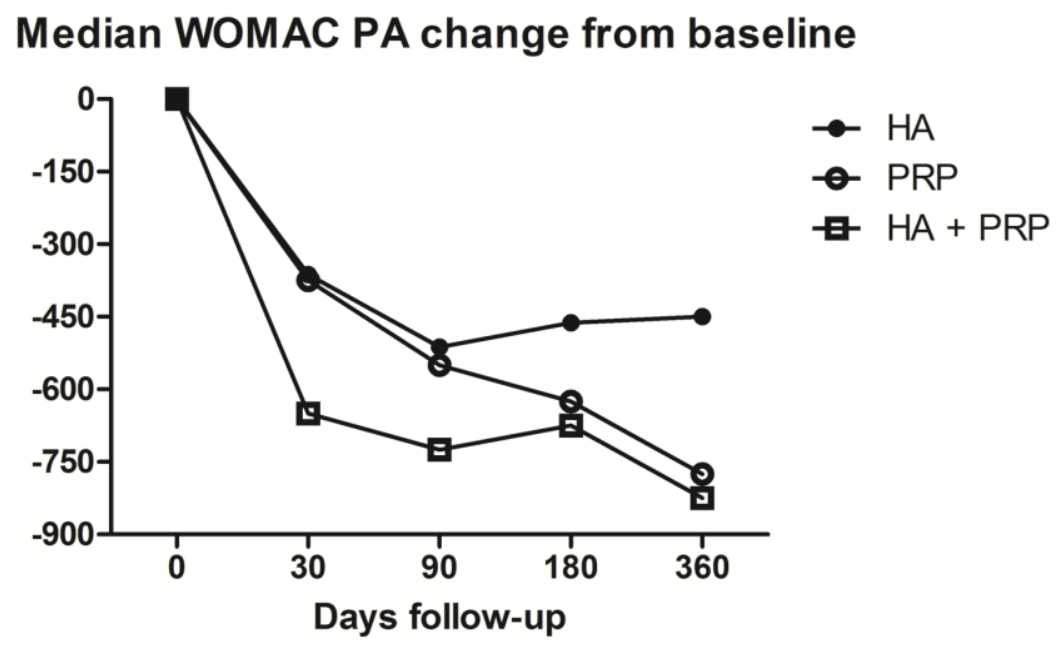

Figure 4: Median WOMAC PA change from baseline 


\section{Discussion}

The use of buffy-coat or leukocyte layer together with PRP was incentivized by studies that highlighted the antimicrobial ${ }^{[25,26]}$ and immune-regulatory ${ }^{[27,28]}$ actions of the leukocytes, as well as proving that the majority of the platelets are found in this layer, together with the leukocytes after the centrifugation ${ }^{[29]}$. There are controversies as to the use of the leukocytes as studies suggests that with the presence of leukocytes, the neutrophils are enabled to liberate metalloproteins that cause degradation to the extracellular matrix and even release free radicals [30]. However, the macrophages are responsible for the removal of the debris, phagocytic function and also have an important role in the balance of the pro-inflammatory and anti-inflammatory aspects of healing. As it is not possible to fractionate the different types of white blood cells, it may be that the absence of macrophages could be more harmful to the cure than any secondary harm inflicted by the presence of the neutrophils. We believe that this increase in CPR levels in the first 90 days of the treatment in groups treated with PRP can be explained due to the increase of inflammatory activity in the joint because of the WBC.

It was furthermore demonstrated that the polymerization and final architecture of the fibrin network considerably influences the intensity and speed of the liberation of growth factors, mainly TGF $\beta 1$ and the presence of the leukocytes maintains a fundamental role in the development of this network ${ }^{[31]}$.

Relative to activation of PRP, the use of chemical activators cause instability in the fibrin network and rapid growth factor release. On the other hand, if the PRP is activated in a more physiological manner, a stable tetramolecular network is formed and it has direct influence in the speed and amount of liberation of the growth factors $^{[31]}$.

Recently, there has been a lot of interest in new treatments aimed at stimulating repair or replacing damaged cartilage in joints. There are currently limited high-level studies in the literature to demonstrate the real efficacy of PRP injections. Sampson et al ${ }^{[32]}$ presented a pilot study involving 14 patients with primary and secondary knee OA where treatment with PRP resulted to significant improvement in function and relief of pain and symptoms. Most of the patients expressed favorable outcome at 12 months after treatment. Kon et $a l^{[33]}$ published a study involving 100 patients with chronic degenerative condition of the knees treated with intraarticular knee injections and followed at 6 and 12 months. They showed statistically significant improvement in all clinical scores and concluded that their preliminary results indicate that treatment with PRP injections is safe and has the potential to reduce pain and improve knee function and quality of life in younger patients with low degree of articular degeneration. Sanchez et $a l^{[34]}$ performed an observational cohort study of 30 patients with knee OA and showed significant reduction of the WOMAC pain subscale at 5 weeks for the autologous preparation rich in growth factors (PRGF) group. They also demonstrated percent reductions in the physical function and overall WOMAC at 5 weeks associated in favor of the PRGF group.

In our study, the patients enrolled have some significant differences between the groups when evaluated the race and comorbidities. In relation to race, it was verified that in the group that used association of PRP and HA, we had more black and less Caucasian people than the other groups. However, in Brazil we have extreme population miscegenation and arthritis does not have relevance in context with race. In relation to comorbidities, it was verified that the group that used PRP + HA had more patients with hypothyroidism and hypertension.

When PRP was compared to HA, the PRP group had significantly greater median VAS improvement at 30, 90, 180, 360 days and significantly greater WOMAC PA improvement at 360 days compared to the HA group. This supports the findings of other studies that showed PRP having superior results versus HA in the treatment of knee OA. Sanchez et al ${ }^{[34]}$ showed that PRP is better in pain, physical activity and overall WOMAC scores in 5 weeks compared to HA. Spakova et $a l^{[10]}$ showed statistically significant better results in the PRP group compared to HA at 3 and 6 month follow up periods in both WOMAC and numeric rating scale (NRS) scores. Kon et $a l^{[35]}$ showed that the PRP group showed better results than the HA group at 6 months follow up in the International Knee Documentation Committee and VAS scores and concluded that autologous PRP injections showed more and longer efficacy than HA injections in reducing pain and symptoms and recovering articular function. Patel et $a l^{[36]}$ evaluated PRP and placebo in treatment of OA using the same methodology as our study with WOMAC and VAS. It was verified that the groups treated with a single or double injection of PRP had an improvement in relation of placebo, however, in 6 months occurred the deterioration of the results. Our study showed an improvement even after 1 year and this can be explained due to one more application and the use of PRP rich in leukocytes.

Many studies have suggested that the application of hyaluronic acid and PRP may have potentially positive effects on cartilage repair and slow down the progression of $\mathrm{OA}^{[9-11]}$. However, to our knowledge, there is lack of studies in literature that examined the combination use of HA and PRP. Studies show that HA provides appropriate matrix and supportive scaffold material for cartilage repair and enhances the mechanical properties of the cartilage ${ }^{[37-39]}$. Thus, it is hypothesized that their combination may be synergistic. According to literature, combining PRP and HA may benefit from their dissimilar biological mechanisms and helping with the signaling molecules as inflammatory molecules, catabolic enzymes, cytokines and growth factors. Also, it was demonstrated that the association of HA+PRP showed synergic effects in the potentials regenerative and antiinflammatory in comparison to HA or PRP alone. This association can alter the inflammatory cytokines in the degeneration process of the chondrocytes through specific mediators (CD44, TGF- $\beta$ RII) and also promote the regeneration of cartilage and inhibit inflammation in OA. The time to all these modifications happen is between 30-90 days after application, which explains the 30-90 days of improvement in WOMAC PA ${ }^{[40,41]}$.

The results of our study showed that the HA + PRP group had statistically significant decrease from baseline of the median VAS and WOMAC PA when compared to the HA group. Thus, combining $\mathrm{HA}$ and PRP resulted to less pain and less functional limitation compared to $\mathrm{HA}$ alone at $30,90,180$ and 360 days.

When comparing HA + PRP group to PRP alone, the combination resulted to a statistically significant improvement in median WOMAC PA values at 30 and 90 days only. The lubrication and support to the extracellular matrix that the HA provided seemed to enable earlier functional benefit to the PRP injection. This combination may result to better rehabilitation and earlier return to activities of daily activities.

The median changes from day 0 baseline in WOMAC pain and WOMAC stiffness were not statistically different among the three groups (HA, PRP and HA+PRP) at any time. No overall WOMAC score comparisons were made.

When the inflammation was evaluated, it was verified that the groups that treated with PRP alone or in combination presented lower levels 
of CPR at the end of follow-up in comparison to HA. Interestingly, in these groups an increase in CPR levels was observed at 90 days. This increase could be explained due to the inflammatory process of cells in the regenerative phase of treatment and also peak of improvement in treatments based in cell therapy.

\section{Study Limitations}

The study utilized self-reported questionnaires such as WOMAC and VAS to assess pain and functional outcomes, which could potentially limit the objectivity of results. In addition, advanced imaging such as MRI was not performed because of high costs, but could have provided more objective data as to the benefit of treatments. Although functional tests can provide more objective responses to treatment, they were not included in the study and should be considered for future studies. Another limitation was the absence of a gold standard or true control group using saline.

\section{Conclusion}

Our results suggest that the use of autologous PRP and its combination with HA are safe and effective methods for treatment of mild to moderate osteoarthritis of the knee. The PRP group had significantly greater reduction in VAS scores at 30, 90, 180 and 360 days and significantly greater WOMAC physical activity improvement at 360 days compared to the HA group. Combining HA and PRP resulted to significantly less pain and less functional limitation compared to HA alone up to 1 year after treatment. HA+ PRP combination also resulted to significantly more physical function early in the treatment (1 month and 3 months) as compared to PRP alone. More randomized controlled studies with larger numbers of patients are needed to confirm these findings and to investigate the persistence of the beneficial effects observed.

\section{References}

1. Conaghan PG, Dickson J, Grant RL, Care and management of osteoarthritis in adults: summary of NICE guidance. BMJ. 2008; 336 (7642): 502-3.

2. Hinton R, Moody RL, Davis AW, Thomas SF. Osteoarthritis: diagnosis and therapeutic considerations. Am. Fam. Physician. 2002; 65 (5): 841-8.

3. Michael JWP, Schlüter-Brust KU, Eysel P.The epidemiology, etiology, diagnosis, and treatment of osteoarthritis of the knee. Dtsch. Arztebl. Int. 2010; 107 (9): 152-62.

4. Gigante A, Callegari L. The role of intra-articular hyaluronan (Sinovial) in the treatment of osteoarthritis. Rheumatol. Int. 2011; 31 (4): 427-44.

5. Bannuru RR, Natov NS, Dasi UR, Schmid CH, McAlindon TE. Therapeutic trajectory following intra- articular hyaluronic acid injection in knee osteoarthritis--meta-analysis. Osteoarthritis Cartilage. $2011 ; 19$ (6):611-9.

6. Bannuru RR, Vaysbrot EE, Sullivan MC, McAlindon TE Relative efficacy of hyaluronic acid in comparison with NSAIDs for knee osteoarthritis: a systematic review and metaanalysis. Semin. Arthritis Rheum. 2014; 43 (5): 593-9.

7. Mishra AK, Skrepnik NV, Edwards SG, Jones GL, Sampson S, Vermillion DA, Ramse y ML, Karli DC, Rettig AC. Efficacy of platelet-rich plasma for chronic tennis elbow: a double-blind, prospective, multicenter, randomized controlled trial of 230 patients. Am J Sport. Med. 2014; 42 (2): 463-71.

8. Patel S, Dhillon MS, Aggarwal S, Marwaha N, Jain A. Treatment with platelet-rich plasma is more effective than placebo for knee osteoarthritis: a prospective, double-blind, randomized tria. Am J Sport. Med. 2013; 41 (2): 356-64.

9. Filardo G, Kon E, Martino AD, Matteo BD, Merli ML, Cenacchi A, Fornasari PM, Marcacci M. Platelet- rich plasma vs hyaluronic acid to treat knee degenerative pathology: study design and preliminary results of a randomized controlled trial. BMC Musculoskelet. Disord.2012; 13 (1): 229.
10. Spaková T, Rosocha J, Lacko M, Harvanová D, Gharaibeh A. Treatment of Knee Joint Osteoarthritis with Autologous PlateletRich Plasma in Comparison with Hyaluronic Acid. Am. J. Phys. Med. Rehabil. 2012; 91(5): 411-7

11. Lubowitz JH, Provencher MT, Poehling GG. Cartilage treatment and biologics current research. Arthroscopy. 2013; 29 (10): 1597-8.

12. Kon E, Mandelbaum B, Buda R, Filardo G, Delcogliano M, Timoncini A, Fornasari PM, Giannini S, Marcacci M. Platelet-rich plasma intra-articular injection versus hyaluronic acid viscosupplementation as treatments for cartilage pathology: from early degeneration to osteoarthritis. Arthroscopy. 2011; 27 (11):1490-501.

13. Spaková T, Rosocha J, Lacko M, Harvanová D, Gharaibeh A. Treatment of knee joint osteoarthritis with autologous platelet-rich plasma in comparison with hyaluronic acid. Am. J. Phys. Med. Rehabil. 2012;91 (5): 411-17.

14. Anitua E, Sánchez M, Nurden AT, Zalduendo MM, Fuente M de la, Azofra J, Andía I. Platelet - released growth factors enhance the secretion of hyaluronic acid and induce hepatocyte growth factor production by synovial fibroblasts from arthritic patients. Rheumatology (Oxford). 2007; 46 (12): 1769-72.

15. KELLGREN JH, LAWRENCE JS. Radiological assessment of osteo-arthrosis. Ann. Rheum. Dis.1957; 16 (4): 494-502.

16. Bellamy N, Buchanan WW, Goldsmith CH, Campbell J, Stitt LW. Validation study of WOMAC: a health status instrument for measuring clinically important patient relevant outcomes to antirheumatic drug therapy in patients with osteoarthritis of the hip or knee. $1988 ; 15(12): 1833-40$.

17. I. MF, Translation and validation of specific quality of life questionnaire for osteoarthritis WOMAC (Western Ontario and McMaster Universities Osteoarthritis Index) for the Portuguese language. 2002.

18. Bellamy N. WOMAC: a 20-year experiential review of a patientcentered self-reported health status questionnaire. J. Rheumatol. 2002; 29 (12): 2473-6.

19. Lei H, Gui L, Xiao R. The effect of anticoagulants on the quality and biological efficacy of platelet- rich plasma. Clin. Biochem. 2009; 42 (13-14): 1452-60.

20. Andrade MGS, de Freitas Brandão CJ, Sá CN, de Bittencourt TCBDSC, Sadigursky M. Evaluation of factors that can modify platelet-rich plasma properties. Oral Surg. Oral Med. Oral Pathol. Oral Radiol. Endod. 2008; 105(1): e5-e12.

21. Perez AGM, Lichy R, Lana JFSD, Rodrigues AA, Luzo ACM, Belangero WD, Santana MHA. Prediction and modulation of platelet recovery by discontinuous centrifugation of whole blood for the preparation of pure platelet-rich plasma. Biores. 2013; 2(4): 307 14.

22. Jacobson M, Fufa D, Abreu EL, Kevy S, Murray MM. Platelets, but not erythrocytes, significantly affect cytokine release and scaffold contraction in a provisional scaffold model. Wound Repair Regen.2008; 16(3): 370-8.

23. Weibrich G, Kleis WKG, Hafner G, Hitzler WE, Wagner W. Comparison of platelet, leukocyte, and growth factor levels in point-of-care platelet-enriched plasma, prepared using a modified Curasan kit, with preparations received from a local blood bank. Clin. Oral Implants Res. 2003; 14 (3): 357-62.

24. Weibrich G, Kleis WKG, Buch R, Hitzler WE, Hafner G. The Harvest Smart PRePTM system versus the Friadent-Schutze plateletrich plasma kit. Comparison of a semiautomatic method with a more complex method for the preparation of platelet concentrates. Clin. Oral Implants Res. 2003;14 (2): 233-39.

25. Cieslik-Bielecka A, Gazdzik TS, Bielecki TM, Cieslik T. Why the platelet-rich gel has antimicrobial activity?. Oral Surg. Oral Med. Oral Pathol. Oral Radiol. Endod. 2007; 103 (3): 303-5; author reply 305-6.

26. Moojen DJ, Everts PA, Schure RM, Overdevest EP, van Zundert A Knape JT, Castelein RM, Creemers LB, Dhert WJ. Antimicrobial activity of platelet-leukocyte gel against Staphylococcus aureus. J Orthop Res. 2008;26(3):404-10.

27. Dohan DM, Choukroun J, Diss A, Dohan SL, Dohan AJJ, Mouhyi J, Gogly B. Platelet-rich fibrin (PRF): a second-generation platelet concentrate. Part III: leucocyte activation: a new feature for platelet concentrates?. Oral Surg. Oral Med. Oral Pathol. Oral Radiol. Endod. 2006; 101 (3): e51-5. 
28. El-Sharkawy H, Kantarci A, Deady J, Hasturk H, Liu H, Alshahat M, Van Dyke TE. Platelet-rich plasma: growth factors and pro- and anti-inflammatory properties. J. Periodontol. 2007; 78(4): 661-9.

29. Weibrich G, Kleis WKG, Hitzler WE, Hafner G. Comparison of the platelet concentrate collection system with the plasma-rich-ingrowth-factors kit to produce platelet-rich plasma: a technical report. Int. J. Oral Maxillofac. Implants. 2005;20(1): 118-23.

30. Toumi H, Best TM. The inflammatory response: friend or enemy for muscle injury? Br. J. Sports Med. 2003;37(4): 284-6.

31. Dohan EDM, Bielecki T, Jimbo R, Barbé G, Del Corso M, Inchingolo F, Sammartino G. Do the fibrin architecture and leukocyte content influence the growth factor release of platelet concentrates? An evidence-based answer comparing a pure plateletrich plasma (P-PRP) gel and a leukocyte- and platelet- rich fibrin (L-PRF). Curr. Pharm. Biotechnol. 2012; 13(7): 1145-52.

32. Sampson S, Reed M, Silvers H, Meng M, Mandelbaum B. Injection of platelet-rich plasma in patients with primary and secondary knee osteoarthritis: a pilot study. Am. J. Phys. Med. Rehabil. 2010; 89(12):961-9.

33. Kon E, Buda R, Filardo G, Di Martino A, Timoncini A, Cenacchi A, Fornasari PM, Giannini S, Marcacci M. Platelet-rich plasma: intra-articular knee injections produced favorable results on degenerative cartilage lesions. Knee Surg. Sports Traumatol. Arthrosc. 2010; 18 (4): 472-9.

34. Sánchez M, Anitua E, Azofra J, Aguirre JJ, Andia I. Intra-articular injection of an autologous preparation rich in growth factors for the treatment of knee OA: a retrospective cohort study. Clin Exp Rheumatol. 2008;26(5):910-3.
35. Kon E, Mandelbaum B, Buda R, Filardo G, Delcogliano M, Timoncini A, Fornasari PM, Giannini S, Marcacci M. Platelet-rich plasma intra-articular injection versus hyaluronic acid viscosupplementation as treatments for cartilage pathology: from early degeneration to osteoarthritis. Arthrosc. J. Arthrosc. Relat. Surg. 2011; 27 (11):1490-1501.

36. Patel S, Dhillon MS, Aggarwal S, Marwaha N, Jain A. Treatment with platelet-rich plasma is more effective than placebo for knee osteoarthritis: a prospective, double-blind, randomized trial. Am. J. Sports Med. 2013: 41(2): 355-64.

37. Levett PA, Hutmacher DW, Malda J, Klein TJ. Hyaluronic acid enhances the mechanical properties of tissue-engineered cartilage constructs. PLoS One. 2014; 9(12): e113216.

38. Ge Z, Li C, Heng BC, Cao G, Yang Z. Functional biomaterials for cartilage regeneration. J. Biomed. Mater. Res. A. 2012; 100 (9): 2526-36.

39. Matsiko A, Levingstone TJ, O'Brien FJ, Gleeson JP. Addition of hyaluronic acid improves cellular infiltration and promotes early-stage chondrogenesis in a collagen-based scaffold for cartilage tissue engineering. $\mathrm{J}$ Mech Behav Biomed Mater. 2012;11:41-52.

40. Andia I, Abate M. Knee osteoarthritis: hyaluronic acid, plateletrich plasma or both in association? Expert Opin Biol Ther. 2014;14(5):635-49.

41. Chen W, Lo W, Hsu W, Wei H, Liu H, Lee C, Chen ST, Shieh Y, Williams DF, Deng W. Synergistic anabolic actions of hyaluronic acid and platelet-rich plasma on cartilage regeneration in osteoarthritis therapy. Biomaterials. 2014;35(36):9599-607.

\author{
Abbreviations \\ PRP: $\quad$ Platelet Rich Plasma \\ HA: Hyaluronic Acid \\ BMC: $\quad$ Bone Marrow Concentrate \\ RBC: $\quad$ Red Blood Cells \\ WBC: White Blood Cells \\ WOMAC: Western Ontario and McMaster Universities Arthritis Index \\ VAS: Visual Analogue Scale \\ BMI: $\quad$ Bone Mass Index
}

\title{
Potential Conflicts of Interests
}

The authors have no conflicts of interest and received no sponsorship for any of the products used in the trial.

\section{Corresponding Author}

Jose F.S.D. Lana, Bone and Cartilage Institute, Instituto do Osso e da Cartilagem (IOC), Avenida Presidente Kennedy, número 1386, Salas 26, 28 e 29, Indaiatuba, São Paulo, Brazil, Zip Code: 13334-170; Email: josefabiolana@ gmail.com 